D. Bachter ${ }^{1}$

S. Goerdt ${ }^{2}$

\section{Präoperative Identifikation und chirurgische Entfernung des Sentinel-Lymphknotens bei malignen Hauttumoren}

Preoperative Identification and Surgical Removal of the Sentinel Lymph Node in Malignant Tumors of the Skin

\section{Zusammenfassung}

Der Sentinel-(= Vorposten)Lymphknoten (SLN) ist ein zuverlässiger Indikator hinsichtlich einer lymphogenen Metastasierung maligner Tumoren. Seine operative Entfernung und anschließende histopathologische Untersuchung ermöglicht ein exaktes Staging der Lymphknoten. Mikrometastasen können zu einem sehr frühen Zeitpunkt der Tumordissemination entdeckt und Hochrisikopatienten gezielt für weitere operative und/oder medikamentöse Therapiemaßnahmen selektioniert werden. Zur Erzielung optimaler Ergebnisse ist eine enge Kooperation von Nuklearmediziner, Operateur und Pathologe unabdingbar. Die präoperative Identifikation muss nach Injektion eines Radiotracers um den Tumor mittels einer dynamischen Lymphabflussszintigraphie erfolgen. Für die chirurgische Entfernung des SLN hat sich die intraoperative Lokalisation mittels einer Szintillationsmesssonde in Kombination mit Patentblauinjektionen bewährt. Die Anwendung geeigneter Schnittserien durch die Lymphknoten und immun-histochemischer Marker erleichtern den histologischen Nachweis der oft sehr kleinen Mikrometastasen erheblich. Mit der beschriebenen Technik gelang die Identifikation und Exstirpation des SLN in nahezu 100\% der Fälle. Während des 6-Jahres-Follow-up entwickelte lediglich ein Patient mit einem negativen SLN Lymphknotenmetastasen. Dies entspricht einer Falschnegativ-Rate von unter $0,3 \%$. Bisher ist noch unklar, ab welcher Tumordicke eine Sentinel Lymphonodektomie (SLNE) indiziert ist. Wir halten die SLNE sowohl bei neu diagnostizierten Melanomen in den klinischen Stadien Ib bis IIIa, als auch bei non-melanozytären high-risk Malignomen bei denen klinisch/ apparativ kein Anhalt für eine bereits bestehende Metastasierung besteht für indiziert. Der Nachweis von Mikrometastasen im SLN ist eine Indikation zur vollständigen Entfernung der re-

\section{Abstract}

The sentinel lymph node is a reliable indicator for a lymphogenous metastasis of malignant tumors. Its operative removal followed by histological examination enables an exact staging of the nodal status. Micrometastases can be detected at a very early time of tumor dissemination and high-risk patients can be selected for further operative and/or medicamentous therapeutic modalities. A close cooperation of surgeon, pathologist and nuclear medicine physician is necessary for optimum results. Preoperative identification must be performed by dynamic lymphoscintigraphy after peritumoral injection of a radiotracer. For the surgical removal of the SLN a combined approach by gammaprobe guidance and blue-dye is the procedure of choice. Complete cutting of the SLN and immunohistochemical stainings facilitate the detection of the often tiny micrometastases. By the described technique the identification and extirpation of the SLN succeded in nearly $100 \%$ of the more than 400 patients. During a 6-year follow-up only one patient with a negative SLN developed a lymph-node recurrence. This corresponds to a false-negative rate of less than $0.3 \%$. However, it is not sure in which tumors a SLNE is indicated. We recommend the SLNE in all new diagnosed melanoma patients in clinical stages Ib to III a and other high-risk skin malignancies. The detection of tumor cells in the SLN is an indication for a radical lymph node dissection, but there is also no consensus concerning this point. Nevertheless studies have to show whether the removal of the SLN is more than a very sensitive diagnostic method or whether patients will profit from this early knowledge of metastases. Hitherto no influence on overall survival can be shown. 
gionären Lymphknoten. Allerdings besteht hierüber noch keine Einigung. Ob die Entfernung des SLN jedoch mehr als ein sehr sensitives diagnostisches Verfahren darstellt oder ob sich für die Patienten Vorteile aus der frühen Kenntnis von Metastasen ergeben, muss in Studien überprüft werden. Auswirkungen auf die Gesamtüberlebenszeit können derzeit noch nicht beantwortet werden.

\section{Einleitung}

Das Konzept des Sentinel-Lymphknotens stellt einen Meilenstein in der klinischen Onkologie dar und ist eine der interessantesten Entwicklungen des letzten Jahrzehnts. Das „lymphatic mapping" mittels dynamischer Lymphabflussszintigraphie hat das Wissen über die Anatomie und Physiologie des Lymphsystems verbessert und trägt so zu einem besseren Verständnis der lymphogenen Metastasierung von Tumoren bei [1]. Die minimal-invasive chirurgische Technik der Sentinel Lymphonodektomie (SLNE) spiegelt den Trend zu einer konservativen operativen Vorgehensweise bei Tumorpatienten wider.

Insbesondere ist es nun möglich, auf die ungezielte prophylaktische Entfernung der regionären Lymphknoten im Sinne einer elektiven Lymphknotendissektion zu verzichten. Dieser morbiditätsträchtige Eingriff wurde über Jahrzehnte bei malignen Hauttumoren unter der Annahme durchgeführt, hierdurch frühzeitig, bei noch geringer Tumorlast, den Metastasierungsweg zu unterbrechen, um möglicherweise eine Lebensverlängerung zu erzielen.

Die SLNE hat sich in der Primärtherapie des malignen Melanoms mittlerweile etabliert $[3,4,8,30]$. Erfahrungen bei anderen Hautmalignomen sind begrenzt. Wie unsere Ergebnisse zeigen, lässt sich diese Technik aber problemlos auf alle lymphogen metastasierenden Tumoren der Haut übertragen [5].

Der SLN ist ein zuverlässiger Prädiktor für das Vorliegen bzw. Fehlen von lymphogenen Metastasen [6,21,25]. Aus seiner Auswertung ergeben sich entscheidende Konsequenzen hinsichtlich der klinischen Stadieneinteilung und weiterführender Therapiemaßnahmen. Zudem sollten die psychologischen Auswirkungen auf den Patienten nicht außer Acht gelassen werden, die durch die Kenntnis von Tumorzellen in den Lymphknoten entstehen. Die Bedeutung des SLN erfordert deshalb die Notwendigkeit von Standards in der Durchführung der SLNE und der sich ergebenden Folgemaßnahmen.

\section{Präoperative Identifilkation des SLN}

Die Identifikation und Definition des SLN erfolgt präoperativ durch eine Lymphabflussszintigraphie. Um eine Bahn im Szintigramm zu sehen und das zeitliche Erscheinen des Lymphknotens festzulegen, benötigt man eine hohe Konzentration des Radiotracers im Lymphgefäßsystem und eine sequenzielle Aufnahmetechnik. Bei malignen Hauttumoren erfolgt nach intrakutaner Injektion des Tracers der Abfluss sehr rasch, so dass bei sofortigem Beginn der Frühaufnahmen post injectionem, gefolgt von Spätaufnahmen, die Bahnen und der SLN frühzeitig sichtbar werden.

Kolloidales Technetium 99m( $\left.{ }^{99 \mathrm{~m}} \mathrm{Tc}\right)$-markiertes Humanalbumin (Nanocoll, Fa. Nycomed, Amersham) oder ${ }^{99 m} T c-Z i n n-(2) S u l f i d$ (Lymphoscint, Fa. Nycomed, Amersham) wird streng intradermal in einem Abstand von $5 \mathrm{~mm}$ um den Tumor bzw. die Vorexzisionsnarbe in Form von 4-12 Aktivitätsdepots injiziert. Die Teilchengröße liegt unter $80 \mathrm{~nm}$, das Injektionsvolumen beträgt weniger als $0,2 \mathrm{ml}$, die Gesamtradioaktivitätsmenge beträgt $80 \mathrm{MBq}$.

Da die Injektionsspritze bei der intrakutanen Injektion unter großem Druck steht, erhöht sich die Kontaminationsgefahr mit Auftauchen von unklaren „hot spots“ in der Nachbarschaft der Injektionsstelle, weshalb diese abgedeckt werden sollte. Außerdem gehen ca. $25 \%$ der zu applizierenden Aktivität durch Austritt aus der Einstichstelle und als Aktivitätsrest in der Spritze verloren.

Unmittelbar nach der Injektion und ca. 1-6 h p.i. werden planare Szintigramme in mind. 2 Ebenen (Sophy-Kamera DSX, LEAP-Kollimator, 100000 counts/Bild oder 10 min Akquisitionszeit, Matrixgröße $128 \times 128$ ) unter Abdeckung der Injektionsstelle angefertigt (Abb.1). Diese Bedingungen sind für die früh- und spätstatischen Aufnahmeserien gleich. In den frühstatischen Aufnahmen sieht man die zu einem oder mehreren Lymphknoten führenden Lymphbahnen. Insbesondere im Kopf-Hals-Bereich muss aufgrund eines schnellen Lymphabflusses und der engen räumlichen Beziehung von Primärtumor und regionärer Lymphknotenstation die Akquisitationszeit pro Bild gelegentlich verkürzt werden, um die sichtbaren Lymphbahnen korrekt zu erfassen. Zudem empfiehlt es sich, das Injektionsvolumen im Bereich des Kopfes auf $<0,1 \mathrm{ml}$ zu reduzieren. Die Frühaufnahmen werden abgebrochen, sobald jede sichtbare Bahn in einen Lymphknoten gemündet hat. Der erste speichernde Lymphknoten, in den eine Abstrombahn mündet, wird als SLN bezeichnet. Führen mehrere Bahnen zu unterschiedlichen Lymphknoten, wird jeder einzelne als SLN angesehen. Diese müssen später alle entfernt werden. In den Spätaufnahmen lassen sich die einzelnen Lymphknoten klar darstellen, die Abstrombahnen sind nicht mehr sichtbar. Sobald der Untersucher eine klare räumliche Vorstel-

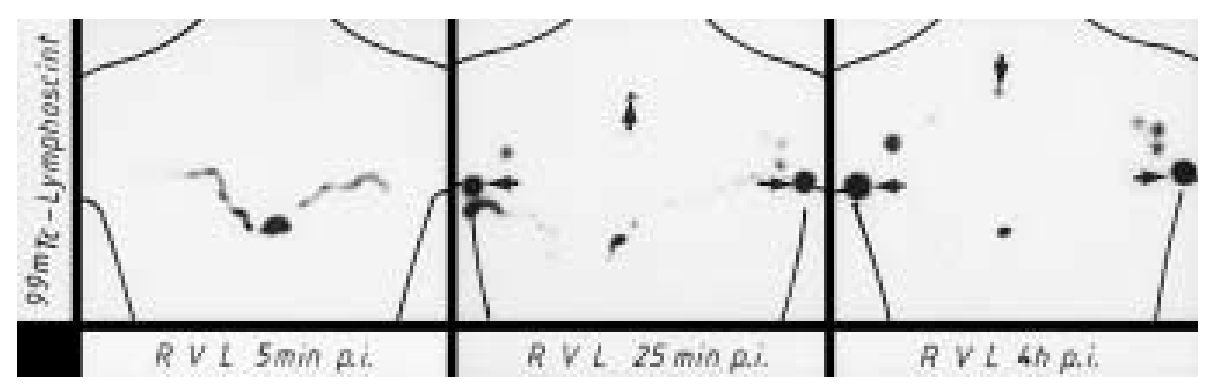

Abb. 1 Lymphabflussszintigramm 
Tab. 1 Charakteristika des SLN im Szintigramm (nach Vogt et al.)

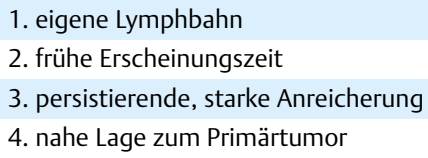

lung von der Lage des SLN hat, wird die Aufnahmeserie beendet. Anschließend ist es zweckmäßig, den SLN mit Hilfe der Szintillationsmesssonde auf der Haut zu markieren. Falls durch die eben beschriebenen Maßnahmen keine eindeutige Identifikation des SLN erreicht werden kann, besteht die Möglichkeit nach einer nochmaligen Injektion von ca. $20 \mathrm{MBq}$ unter verbesserter Positionierung des Patienten den Abtransport des Radiotracers zu kontrollieren. Die Charakteristika des SLN im Szintigramm sind aus Tab. 1 ersichtlich [34].

Erfolgt die Entfernung des SLN am Tage nach der Lymphabflussszintigraphie, ist keine Nachinjektion des Radionuklids erforderlich, da die gespeicherte Radioaktivitätsmenge ausreicht, um den SLN intraoperativ mit der Gammasonde zu lokalisieren. Bei zu erwartenden längeren Zeitintervallen wird die präoperative Lymphabflussszintigraphie mit ca. $40 \mathrm{Mbq}{ }^{99 \mathrm{~m} T c-N a n o k o l l o i d}$ durchgeführt und es erfolgt am Operationstag mind. 1 h präoperativ eine Zweitinjektion mit einer analogen Radioaktivitätsmenge.

\section{Intraoperative Lokalisationsdiagnostik}

Grundsätzlich halten wir ein 2-Tages-Protokoll für sinnvoll. Dies bedeutet Lymphabflussszintigraphie am Tag 1, chirurgische Entfernung des SLN am darauffolgenden Tag 2. Um den SLN intraoperativ aufzufinden, gibt es prinzipiell zwei Möglichkeiten. Die Kombination beider Techniken ist am günstigsten.

\section{Farbstoffmethode}

Morton [22], der das Konzept des SLN erstmals beim malignen Melanom beschrieb, benutzte zunächst Patentblau V zur intraoperativen Darstellung des SLN.

0,5-1,0 ml 2,5\%iges Patentblau V (2,4-Disulfo-5-Hydroxy-4',4"bis (diäthylamino)-triphenylcarbinolmononatriumsalz) wird um den verdächtigen Hauttumor bzw., sofern dieser bereits exzidiert worden ist, um die Vorexzisionsnarbe streng intradermal injiziert. Anschließend wird über der drainierenden Lymphknotenstation, die tags zuvor mittels einer Lymphabflussszintigraphie dargestellt wurde, inzidiert und ein entsprechend großer Hautlappen gehoben. Dabei ist zu beachten, dass das Zeitfenster in dem der SLN eine Blaufärbung aufweist, begrenzt ist. Bei dieser Methode muss also die SLNE vor der Exzision des Primärtumors bzw. der Nachexzision durchgeführt werden. Unter sorgfältiger Präparation in die Tiefe, möglichst unter Belassen der ebenfalls blau gefärbten Lymphbahnen sucht man den blau angefärbten SLN auf (Abb. 2). Da der Farbstoff sehr schnell wieder aus den Lymphknoten verschwindet, muss gelegentlich nachinjiziert werden. Dies hat bei entsprechender Lage des Primärtumors, z.B. am Rücken, unter Umständen mehrere Umlagerungen zur Folge. Der SLN wird dann unter sorgfältiger Ligatur der zu- und

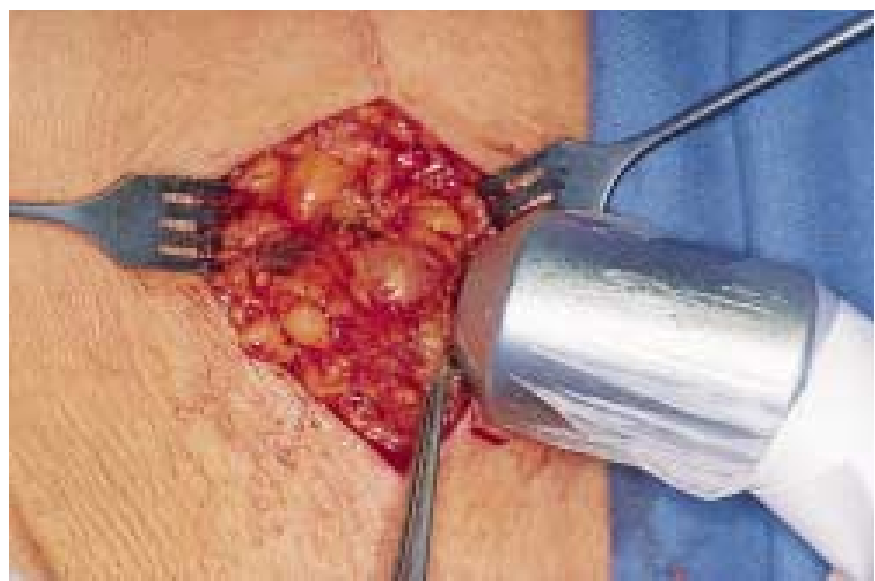

Abb. 2 Operationssitus mit Patentblau.

abführenden Lymphbahnen im Ganzen exstirpiert und sogleich in Formalin eingelegt. Sind im Szintigramm mehrere SLN beschrieben, müssen alle in eben beschriebener Weise entfernt werden. Anschließend wird der Defekt schichtweise verschlossen, ggf. unter Einlage einer Redonsaugdrainage. Heutzutage kann die Entfernung des SLN mit der Farbstoffmethode allein nicht mehr empfohlen werden, da sie einige Nachteile gegenüber der Gammasondentechnik aufweist.

\section{Gammasondentechnik}

1993 beschrieben Alex und Krag [3] erstmals die Markierung des SLN mit einem Radiotracer und die nachfolgende intraoperative Lokalisation mittels einer Gammasonde.

Diese Technik ist wesentlich einfacher zu handhaben, bietet im Vergleich zur Farbstoffmethode viele Vorteile und weist bei fachgerechter Anwendung eine Sensitivität von nahezu 100\% auf.

Die am Vortag zur Anfertigung des Lymphabflussszintigramms injizierte Menge des Tracers (i. d. R. $80 \mathrm{MBq}$ ) reicht in der Regel aus, um mittels Radioaktivitätsmessungen den SLN während der am nächsten Tag stattfindenden Operation zu lokalisieren. Mittlerweile existieren verschiedene Sondensysteme unterschiedlicher Hersteller. Es ist für die Reproduzierbarkeit der Methode unabdingbar, eine Sonde zu verwenden, die die Qualitätskriterien bezüglich Ortsselektivität, Nachweisempfindlichkeit, Abschirmung, Energiediskriminierung und Handhabbarkeit erfüllt (Details s. [35]).

\section{Malignes Melanom}

Wir empfehlen bei Verdacht auf ein malignes Melanom folgendes Vorgehen (Abb.3). Bei In-situ-Tumoren wird im ersten Schritt der verdächtige Hauttumor inklusive des peritumoralen Radioaktivitätsdepots mit einem Sicherheitsabstand von $1 \mathrm{~cm}$ nach allen Seiten, tiefenwärts bis auf Faszienebene exzidiert und sogleich zur histologischen intraoperativen Schnellschnittuntersuchung abgegeben. Dieser Eingriff kann in jedem Fall in Lokalanästhesie erfolgen. Bei vorliegender Diagnose entfällt dieser Schritt. Da Nachexzision und SLNE i. A. in Allgemeinanästhesie durchgeführt werden, sollte der Patient bereits im Vorfeld für eine Intubationsnarkose vorbereitet werden und kann sofort nach Erhalt des Schnellschnittergebnisses in das Op-Programm eingeschleust werden. 


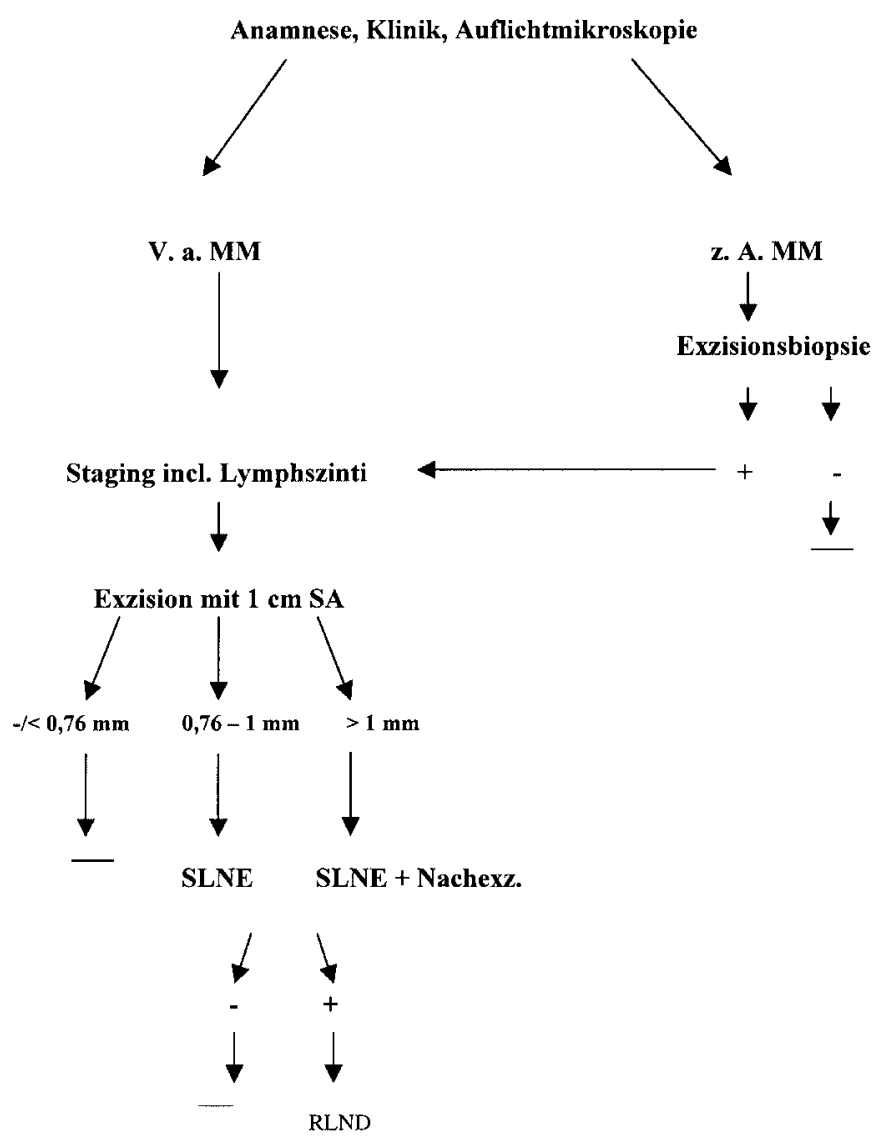

Abb. 3 Flussschema malignes Melanom.

Im Falle einer Bestätigung des Malignitätsverdachtes und bei einem vertikalen Tumordurchmesser nach Breslow von über $0,75 \mathrm{~mm}$, wird der Patient in das SLNE-Protokoll eingeschleust. Zunächst wird die Primärexzisionsstelle tumordickenabhängig, also ggf. nach weiterer Exzision mit den in den DDG-Richtlinien [24] empfohlenen Sicherheitsabständen und anschließender plastischer Deckung, versorgt.

Im nächsten Schritt wurde mit einer für ${ }^{99 \mathrm{~m}} \mathrm{Tc}$ optimierten und kollimierten Szintillationsmesssonde (C-Trak ${ }^{\circledR}$-System, Fa. CareWise, Morgan Hill, Kalifornien) der SLN von außen aufgesucht. Hierbei leistet die tags zuvor angebrachte Hautmarkierung eine wertvolle Orientierungshilfe. Im Bereich des Aktivitätsmaximums wird eine kleine Hautinzision angelegt und der Lymphknoten unter Führung der Gammasonde sorgfältig unter Ligatur der zu- und abführenden Lymphbahnen exstirpiert (Abb. 4). Erst wenn durch Aktivitätsmessungen im Operationsgebiet und am Exstirpationspräparat die Vollständigkeit der Entfernung überprüft worden ist, erfolgt der schichtweise Verschluss der Wunde, wobei ggf. eine Saugdrainage eingelegt werden kann. In der Regel weist der SLN die höchste Radioaktivität auf.

Gelegentlich wird ein Konglomerat aus mehreren Lymphknoten entfernt. In diesem Fall werden sämtliche entfernten Knoten auf ihren Radioaktivitätsgehalt hin überprüft, durchnummeriert und der eigentliche SLN mit einem Faden markiert. Anschließend werden sie in Formalin eingelegt.

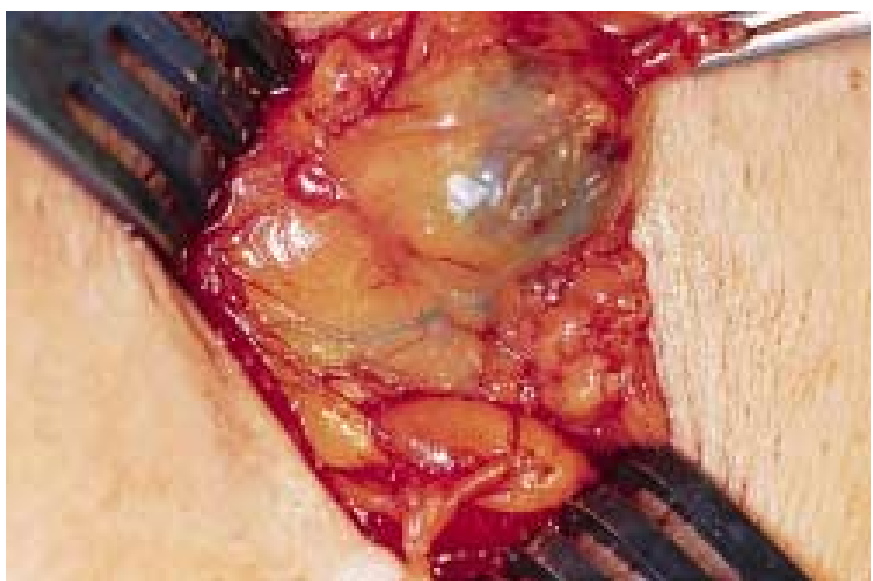

Abb. 4 Operationssitus mit Gammasonde.

\section{Nicht-melanozytäre Hautmalignome}

Bei V. a. einen nicht-melanozytären malignen Hauttumor wird in der Regel zunächst eine 4-mm-Stanzbiopsie zur Diagnosesicherung entnommen. Erst nach Erhalt des histologischen Untersuchungsergebnisses wird anhand einer Risikoabschätzung hinsichtlich der Wahrscheinlichkeit einer lymphogenen Metastasierung entschieden, ob der Patient in das SLNE-Protokoll eingeschleust wird. Ansonsten entspricht das Vorgehen dem oben Beschriebenen.

\section{Histologische Untersuchung des Sentinel-Lymphknotens}

Nähere Details in diesem Heft. In Kürze: Nach eintägiger Formalinfixierung wird der Lymphknoten weiterverarbeitet. Er wird in max. $1 \mathrm{~mm}$ dünne Scheiben entlang der Längsachse vollständig auflamelliert. Von jedem Schnitt werden neben Hämatoxy-Eosin, immunhistochemische Färbungen entsprechend der Tumorentität angefertigt. Damit lassen sich Mikrometastasen leichter entdecken [12]. Werden im SLN Tumorzellen nachgewiesen, so erfolgt in einer zweiten Operation die radikale Ausräumung der entsprechenden Lymphknotenregion. Die entnommenen Dissektate werden in eben beschriebener Weise histologisch aufgearbeitet.

\section{Ergebnisse}

Betrachtet man die Ergebnisse verschiedener Arbeitsgruppen (Tab. 2), so wird deutlich, dass die Entfernung des SLN beim malignen Melanom eine Methode mit hoher Sensitivität darstellt. Mit der intraoperativen Detektion mittels Gammasonde lassen sich Erfolgsraten bis 100\% erzielen. Dagegen bringt die Farbstofftechnik deutlich schlechtere Resultate (82-99\%). Der Anteil an Patienten mit Metastasen im SLN schwankt von $12-23 \%$. Die Falschnegativ-Rate liegt im Bereich von 0-2\%.

Über die Anwendung der Sentinel-node-Technik bei nicht-melanozytären malignen Hauttumoren gibt es bisher kaum größere Untersuchungen. Unsere Erfahrungen bei 37 Patienten mit unterschiedlichen Hautmalignomen bestätigen die Ergebnisse, die hinsichtlich der Durchführbarkeit beim malignen Melanom beschrieben werden (Sensitivität 100\%, Falschnegativ-Rate 0\%) [5]. 
Tab. 2 Ergebnisse der SLNE beim malignen Melanom

\begin{tabular}{lllllll}
\hline Autor & Zahl & Indikation & Methode & SLN identif. (\%) & SLN+ (\%) & falsch neg. (\%) \\
\hline Morton & 223 & Klin. Stad. I, II & Farbstoff & 82 & 21 & 1 \\
\hline Thompson & 118 & Klin. Stad. I, II & Farbstoff & 87 & 21 & 0 \\
\hline Glass & 132 & $>0,75$ mm & Farbstoff & 99 & 23 & nicht best. \\
\hline Krag & 121 & Klin. Stad. I, II & Gamma & 98 & 12 & 0 \\
Albertini & 106 & $>0,75$ mm & beide & 96 & 15 & 0 \\
\hline Pijpers & 135 & Klin. Stad. I, II & beide & 100 & 21 & 0 \\
\hline Bachter & 412 & Klin. Stad. I, II & beide & 99 & 21 & 0,3 \\
\hline
\end{tabular}

\section{Diskussion}

Die Entfernung des Sentinel-Lymphknotens führte zu einem grundlegenden Wandel im operativen Tumormanagement [26]. Die SLNE ersetzt die ELND, welche über Jahrzehnte insbesondere beim malignen Melanom sehr kontrovers diskutiert worden war, letztendlich aber den eindeutigen Beweis eines Benefits schuldig geblieben ist $[9,10,28,33]$. Wenngleich in einigen retrospektiven Studien bestimmte Untergruppen von Melanompatienten davon zu profitieren schienen $[11,20]$, so lässt es sich nicht leugnen, dass ein Großteil der Patienten einer unnötigen Operation mit einem hohen Morbiditätsrisiko unterzogen worden war [32]. Auch bei potenziell lymphogen metastasierenden nicht-melanozytären Malignomen gehörte und gehört eine ELND häufig zum Standardrepertoire in der operativen Behandlung. Aus unserer Sicht ist sie auch hier nicht mehr zeitgemäß, da wir mit der SLNE nun ein minimal invasives Operationsverfahren zur Hand haben, welches ein exaktes Staging der dem Primärtumor zugehörigen Lymphknotenregion ermöglicht. Diese Methode reserviert die frühzeitige Entfernung der regionären Lymphknoten gezielt den Patienten mit klinisch okkulten Lymphknotenmetastasen.

Obwohl diese Operationstechnik auf den ersten Blick einfach scheint, muss gerade im Hinblick auf die Bedeutung, die dem SLN zugemessen wird, eine Standardisierung in der präoperativen Identifikation und der chirurgischen Entfernung gewährleistet sein.

Entscheidend für den Erfolg dieser Methode ist eine enge Zusammenarbeit zwischen Nuklearmediziner und Operateur. Wir bevorzugen ein 2-Tages-Protokoll, weil hierdurch eine optimale präoperative Diagnostik ohne Zeitdruck gewährleistet wird, die wiederum eine Zeitersparnis in Bezug auf die Operationsdauer mit sich bringt. Es hat sich bewährt, dass entweder der Nuklearmediziner bei der Operation mit am Tisch steht oder dass der Operateur bei der Lymphabflussszintigraphie anwesend ist. Insbesondere in schwierigen anatomischen Regionen wie z.B. im Kopf-Hals-Gebiet kann die präoperative Identifikation und das nachfolgende intraoperative Auffinden schwierig sein, weshalb genaue Kenntnisse des Lymphabflusses entscheidend sind $[1,23]$.

Grundsätzlich muss die präoperative Identifikation mittels einer dynamischen Lymphabflussszintigraphie erfolgen $[18,34]$. Die frühstatischen Aufnahmen müssen unmittelbar nach der Injektion beginnen. Die zum Lymphknoten führende Bahn muss so lange verfolgt werden, bis sie in einen Lymphknoten mündet. Dieser ist per definitionem der SLN. Tauchen mehrere Bahnen auf, muss jede bis zu ihrem Endpunkt verfolgt werden, so dass u. U. mehrere SLN innerhalb einer Lymphknotenregion existieren können. Ebenso gibt es, abhängig vom Sitz des Primärtumors gelegentlich mehrere SLN in unterschiedlichen Regionen. Entscheidend ist, dass in jedem Fall alle SLN entfernt werden.

Mit zunehmender Dauer ab Injektionszeitpunkt wird der Radiotracer auch in weiter proximal gelegene Lymphknoten transportiert. Die Sensitivität der Lokalisationsdiagnostik litt darunter aber keinesfalls, da intraoperativ das Radioaktivitätsverhältnis von SLN zu den weiter proximal liegenden Lymphknoten immer dem der präoperativen Voruntersuchung entsprach.

Liegt der Primärtumor in unmittelbarer Nähe zur drainierenden Lymphknotenstation, kann die Identifikation problematisch sein. Der SLN kann durch das Radioaktivitätsdepot der Injektionsstelle überlagert werden und es können nachgeschaltete Lymphknoten erscheinen, die bei nicht sichtbaren Bahnen nicht vom SLN differenziert werden können. Lösungsmöglichkeiten bestehen in einer Reduktion des Injektionsvolumens bei gleichbleibender Radioaktivitätsmenge, da hierdurch eine geringere Verteilung in das umliegende Gewebe resultiert und einer Verkürzung der Akquisitionszeit pro Aufnahme [31,34].

Von Bedeutung scheint auch die Art des verwendeten Radiotracers zu sein [16]. Zu Beginn verwendeten wir ${ }^{99 m} \mathrm{Tc}-Z i n n(2)-S u l-$ fid. Obgleich der Hersteller eine Teilchengröße von unter $80 \mathrm{~nm}$ für das von uns verwendete Schwefelkolloid angibt, könnte dieses nach Erhitzen der Lösung in wesentlich größeren Einheiten vorgelegen haben. Es ist bekannt, dass sich große Partikel langsam bzw. überhaupt nicht von der Injektionsstelle bewegen, während sehr kleine Partikel rasch abtransportiert werden. Krag [17] gibt eine 10\%ige Versagerquote des SLN-Nachweises bei der Verwendung von Schwefelkolloid an. Wir konnten diese hohe Fehlerquote nicht bestätigen, lediglich bei 3 Patienten fand kein Lymphabfluss statt. Möglicherweise liegt dies an der Tatsache, dass es sich bei ihm meist um voroperierte Patienten handelte. Neuerdings verwenden wir zur Lymphabstromszintigraphie kolloidales Tc-markiertes Humanalbumin, das hinsichtlich der Partikelgröße die besser definierte Substanz zu sein scheint. Damit traten in der Folgezeit keine derartigen Probleme mehr auf, was natürlich auch an der zunehmenden Erfahrung in der Diagnostik begründet sein kann und nicht unbedingt auf die Tracereigenschaften zurückzuführen sein muss. 
Ein bisher noch nicht eindeutig einordenbares Problem stellen sog. Intervalllymphknoten dar [25]. Hierbei handelt es sich um Anreicherungen, die auf der Strecke zwischen Primärtumor und regionärer Lymphknotenstation liegen. In solchen Fällen müssen kurzzeitige Speicherungen, welche nach wenigen Stunden wieder verschwinden, von wahren SLN unterschieden werden. Beide kommen gehäuft bei voroperierten Patienten vor. Bei ersteren handelt es sich möglicherweise lediglich um lakunenartige Erweiterungen von Lymphgefäßen oder um zwischengeschaltete Lymphknoten, die keine Filterfunktion im eigentlichen Sinne besitzen. In einigen Fällen fanden wir SLN in der Kniekehle bzw. Ellenbeuge. Die Bestätigung der Richtigkeit wurde in einem Fall durch das Vorhandensein von Tumorzellen bestätigt. Schwieriger wird die daraus resultierende Konsequenz des weitergehenden chirurgischen Vorgehens hinsichtlich der Entscheidung wo die radikale Dissektion durchgeführt werden soll, also ob in derartigen Fällen die nächste Lymphknotenstation disseziert werden sollte.

In der intraoperativen Lokalisation des SLN hat die Farbstofftechnik als alleinige Methode ausgedient, da sie deutliche Nachteile gegenüber dem gammasondengesteuerten Verfahren aufweist (Tab.3). Sie ist in der Kombination hilfreich, da die Blauanfärbung der Lymphknoten deren intraoperatives Auffinden erleichtert und dadurch die Operationszeit verkürzt. Der SLN färbt sich nicht in allen Fällen mit Patentblau an [22], woraus eine geringere Sensitivität resultiert [14]. Insbesondere bei Tumorlokalisationen am Rumpf und im Gesichts-/Hals-Bereich ist die Versagerquote relativ hoch. Der Operateur benötigt große Erfahrung, es ist eine größere Anzahl von Eingriffen nötig, um diese Technik zu beherrschen, die Lernkurve ist länger als bei der SLNE mittels einer Gammasonde. Zum Auffinden des SLN müssen längere Inzisionen angelegt werden und oft ist es notwendig, größere Hautlappen zu heben, um eine ausreichende Übersicht über den Operationssitus zu bekommen. Hieraus ergibt sich eine stärkere Gewebstraumatisierung, die Operationszeit ist länger, die kosmetischen Ergebnisse unschöner. Da der Farbstoff zudem sehr schnell wieder aus den Lymphknoten verschwindet, muss gelegentlich nachinjiziert werden. Dies hat bei entsprechender Lage des Primärtumors, z. B. am Rücken, unter Umständen mehrere Umlagerungen zur Folge. Seltene Komplikationen sind permanente Farbstofftätowierungen, die wir bisher zweimal beobachteten, des Weiteren Einzelfallberichte über allergische Reaktionen auf den Farbstoff [15]. Im Kopf-Hals-Bereich verzichten wir auf die Injektion von Patentblau, da einerseits der Operationssitus unübersichtlich wird, da der Farbstoff auch in tiefere

Tab. 3 Nachteile der Farbstoffmethode zur intraoperativen SLNLokalisation

\footnotetext{
1. geringere Sensitivität - SLN färbt sich nicht immer blau an

2. längere Lernkurve des Operateurs

3. größere Inzisionen nötig, stärkere Gewebstraumatisierung

4. zeitaufwändiger

5. enges Zeitfenster der Farbstoffanreicherung, evtl. Nachinjektionen und Umlagern des Patienten notwendig

6. unübersichtliches Operationsgebiet im Bereich der Injektionsstelle, insbesondere im Gesicht problematisch

7. permanente Farbstofftätowierungen

8. allergische Reaktionen auf Patentblau (sehr selten)
}

Schichten diffundiert und dort die Gefahr wichtige Strukturen zu verletzen größer ist. Zudem kann das Risiko einer permanenten Farbstofftätowierung in diesem ästhetisch exponierten Bereich nicht in Kauf genommen werden. Obligat ist nach der Farbstoffinjektion eine Grünverfärbung des Urins, was zu großer Aufregung bei Patient und medizinischem Personal führen kann. Einmal wurde bei einer schlanken blassen Patientin eine Blauverfärbung des gesamten Integuments beobachtet.

Alle mit Patentblau angefärbten SLN speicherten auch das Radionuklid. Zusätzliche blaue, aber nicht radioaktiv markierte Lymphknoten fanden sich nie. Dies deckt sich auch mit den Ergebnissen anderer Arbeitsgruppen [2,25].

Die Exstirpation unter Führung einer Gammasonde ist sehr schnell erlernbar und unter Beachtung der Besonderheiten einfach durchzuführen $[2-4,13,23]$. Durch die exakte Ortung können Richtungsabweichungen bei der Präparation selbst in schwierigen anatomischen Regionen korrigiert werden, so dass eine äußerst gewebeschonende Entfernung möglich wird. Die Inzisionen können sehr klein gehalten werden, wodurch hervorragende kosmetische Ergebnisse erzielt werden können. Der Eingriff kann sogar in Lokalanästhesie erfolgen. Wir konnten den SLN bis auf zwei Ausnahmen problemlos lokalisieren und entfernen. Bei beiden Versagern handelte es sich um Primärtumoren am Hals, wo sich der SLN in der Supraklavikulargrube darstellte. Aufgrund eines sehr hohen Backgrounds der Injektionsstelle konnte intraoperativ die umschriebene Anreicherung des SLN nicht differenziert werden. Ebenfalls nicht unbedeutend ist die Beschaffenheit der verwendeten Gammasonde [35]. Sie sollte den beschriebenen Kriterien entsprechen. Insbesondere sollte die Möglichkeit zur Änderung der Ortsselektivität, z. B. durch verschiedene Kollimatoraufsätze, gegeben sein, da hierdurch in schwierigen anatomischen Regionen eine zielgerichtete Präparation unter Schonung von Nerven und Gefäßen möglich wird [18]. Nennenswerte Komplikationen traten nicht auf. Gelegentlich kam es postoperativ zu kleinen Seromen bzw. Hämatomen, die jedoch mit den üblichen Therapiemodalitäten gut beherrschbar waren.

Eine immer wieder gestellte Frage ist die Strahlenexposition der Operateure und der Pathologen. Im Rahmen der SLNE ist die Strahlenbelastung für den Chirurgen am höchsten [7]. $24 \mathrm{~h}$ nach Injektion von $40 \mathrm{MBq}$ Nanokolloid liegt die Dosisleistung in $0,5 \mathrm{~m}$ Abstand von der Injektionsstelle bei ca. $0,1 \mu \mathrm{Sv} / \mathrm{h}$. Berücksichtigt man diese Werte und Stellungnahme der Strahlenschutzkommission, so kann abgeschätzt werden, dass sowohl für das Op-Personal als auch für die Mitarbeiter in der Pathologie die Strahlenexposition von $1 \mathrm{mSv} / \mathrm{Jahr}$ nicht erreicht werden kann. Es sind folglich keine zusätzlichen Strahlenschutzmaßnahmen notwendig.

Obwohl die SLNE eine sehr sensitive und dabei komplikationsarme Operationstechnik darstellt, muss man überlegen, bei welchen Patienten ihr Einsatz sinnvoll ist, zumal sie mit einem beträchtlichen Personal- und Kostenaufwand verbunden ist. Lokalrezidive nach SLNE treten selten auf $(0-1,5 \%)[6,17,25]$. In unserem Kollektiv entwickelte ein Patient von $324(0,3 \%)$ eine Lymphknotenmetastase in der Leiste nach SLNE mit negativem SLN. Eine Erklärungsmöglichkeit könnte sein, dass dieser Patient am Unterschenkel bereits voroperiert war. Dadurch könnten sich 
die Verhältnisse des Lymphabflusses geändert haben und ein falscher Lymphknoten angesteuert worden sein, der irrtümlich für den SLN gehalten wurde.

In der Primärtherapie des malignen Melanoms wird von den meisten Autoren die SLNE ab einer Tumordicke von $1 \mathrm{~mm}$ empfohlen. In unserem Kollektiv fand sich kein positiver SLN bei Patienten im Stadium Ia. Dies deckt sich mit den Ergebnissen von Pijpers et al. [25]. Krag et al. [17] fanden lediglich bei einem von 24 Patienten mit dünnen Melanomen einen SLN mit Tumorzellen. Deshalb ist aus unserer Sicht die SLNE bei Melanomen im klinischen Stadium Ia verzichtbar. Da wir in einigen Fällen auch im Bereich zwischen 0,75 und $1 \mathrm{~mm}$ positive SLN fanden, entschlossen wir uns ab Stadium Ib zur SLNE. Letztendlich ist es aber noch unklar, ab welcher Tumordicke eine SLNE indiziert ist. Die Grenzen schwanken erheblich und liegen im Bereich von 0,75-1,5 mm. Da insbesondere in den USA der Invasionslevel nach Clark für die Stadieneinteilung wichtig ist, sind die Ergebnisse der einzelnen Arbeiten nicht unbedingt vergleichbar.

Eine weitere, immer wieder aufgeworfene Frage ist, ob die SLNE als therapeutische Maßnahme angesehen werden kann. In den Dissektaten nach konsekutiver radikaler Lymphknotenausräumung der befallenen Region bei positivem SLN fanden sich in ca. $25 \%$ zusätzliche Mikrometastasen, abhängig vom Ausmaß des Tumorbefalls im SLN. In der Literatur schwanken die Angaben diesbezüglich von $13-50 \%[10,14,26]$. Ein positiver SLN ist eine hypothetische Indikation zur RLND, zumal bis heute keine gesicherten adjuvanten Therapien zur Eradikation von Melanomzellen existieren, wenngleich der Nutzen einer derartigen Operation aber noch nicht bewiesen werden konnte.

Hinsichtlich nicht-melanozytärer Hautmalignome können die Erfahrungen, die wir beim Melanom gemacht haben, in Bezug auf Sensitivität und Durchführbarkeit bestätigt werden [5]. Natürlich sollte die Methode nur bei ausgewählten Hochrisikopatienten für eine lymphogene Metastasierung zur Anwendung kommen, da in der Zwischenzeit ökonomische Gesichtspunkte eine zunehmende Rolle spielen. Grundsätzlich muss diese Operationstechnik zum gegenwärtigen Zeitpunkt bei nicht-melanozytären Hauttumoren als experimentell eingestuft werden, da bisher größere Untersuchungen über homogene Patientenkollektive fehlen.

Zusammenfassend kann gesagt werden, dass die SLNE einen Meilenstein in der Tumorchirurgie darstellt. Diese Technik erlaubt ein exaktes Staging und reserviert die frühzeitige Entfernung der regionären Lymphknoten gezielt den Patienten mit nachgewiesenen Lymphknotenmetastasen. Die ELND wird dadurch obsolet. Ihre Wertigkeit bezüglich der Gesamtüberlebenszeit muss trotzdem noch weiter evaluiert werden.

\section{Literatur}

${ }^{1}$ Alazraki N, Eshima D, Eshima L et al. Lymphoscintigraphy, the sentinel node concept, and the intraoperative gamma probe in melanoma, breast cancer and other potential cancers. Sem Nucl Med 1997; 27: $55-67$

${ }^{2}$ Albertini JJ, Cruse CW, Rapaport D et al. Intraoperative radio-lymphoscintigraphy improves sentinel lymph node identification for patients with melanoma. Ann Surg 1996; 223: 217-224
${ }^{3}$ Alex JC, Krag DN. Gamma-probe-guided localization of lymph nodes. Surg Oncol 1993; 2: 137-144

${ }^{4}$ Bachter D, Balda BR, Vogt H, Büchels H. Die „sentinel“-Lymphonodektomie mittels Szintillationsdetektor. Hautarzt 1996; 47: 754 - 758

${ }^{5}$ Bachter D. Ist die Entfernung des Sentinel Lymphknotens bei nichtmelanozytären Hautmalignomen sinnvoll? In: R. Rompel, J. Petres (Hrsg). Fortschritte der Operativen und Onkologischen Dermatologie, Bd. 18. Im Druck. 2002

${ }^{6}$ Bachter D, Michl C, Büchels H, Vogt H, Balda BR. The predictive value of the sentinel lymph node in malignant melanomas. Recent Results Cancer Res 2001; 158: 129-136

${ }^{7}$ Bares R, Müller B, Fas J et al. The radiation dose to surgical personnel during intraoperative radioimmunoscintimetry. Eur J Nucl Med 1992; 19: $110-112$

${ }^{8}$ Büchels H, Vogt H, Bachter D. Szintillationsgesteuerte SentinelLymphadenektomie beim malignen Melanom. Chirurg 1997; 68: 45-50

${ }^{9}$ Balch CM. The role of elective lymph node dissection in melanoma: rationale, results and controversies. J Clin Oncol 1988; 6: 163-172

${ }^{10}$ Balch CM, Milton GW, Cascinelli N, Sim FH. Elective lymph node dissection: pros and cons. In: Balch CM, Houghton AN, Milton GW, Sober AJ, Soong SJ (Eds). Cutaneous melanoma, 2nd ed. Philadelphia: Lippincott, 1992: 345-366

${ }^{11}$ Balch CM, Soong SJ, Bartolucci AA et al. Efficacy of an regional elective lymph node dissection of 1 to $4 \mathrm{~mm}$ thick melanomas for patients 60 years of age and younger. Ann Surg 1996; 224: 255-266

12 Cochran AJ, Wen DR, Morton DL. Occult tumor cells in the lymph nodes of patients with pathological stage I malignant melanoma: an immunohistochemical study. Am J Surg Path 1988; 12: 612 -618

${ }^{13}$ Glass FL, Messina JL, Cruse W et al. The use of intraoperative radiolymphoscintigraphy for sentinel node biopsy in patients with malignant melanoma. Dermatol Surg 1996; 22: 715-720

${ }^{14}$ Godellas CV, Berman CG, Lyman G et al. The identification and mapping of melanoma regional nodal metastases: Minimal invasive surgery for the diagnosis of nodal metastases. Am Surg 1995; 61: 97-101

${ }^{15}$ Hietala SO, Hirsch JI, Faunce HP. Allergic reaction to patent blue violet during lymphography. Lymphology 1977; 10: 158-160

${ }^{16}$ Johansson L, Mattson S, Nosslin B et al. Effective dose from radiopharmaceuticals. J Nucl Med 1992; 19: 933 -938

${ }^{17}$ Krag DN, Meijer SJ, Weaver DL. Minimal-access surgery for staging of malignant melanoma. Arch Surg 1995; 130: 654-658

${ }^{18}$ Leong SPL, Steinmetz I, Habib FA et al. Optimal selective sentinel lymph node dissection in primary malignant melanoma. Arch Surg 1997; 132: 666-673

${ }^{19}$ Miliotes G, Albertini J, Berman C et al. The tumor biology of melanoma nodal metastases. Am Surg 1996; 62: 81 - 88

${ }^{20}$ Milton GW, Saw HM, McCarthy WH et al. Prophylactic lymph node dissection in clinical stage I cutaneous malignant melanoma: results of surgical treatment in 1319 patients. Br J Surg 1982; 69: 108 - 111

${ }^{21}$ Morton DL, Wen DR, Cochran AJ. Management of early-stage melanoma by intraoperative lymphatic mapping and selective lymphadenectomy. An alternative to routine elective lymphadenectomy or „watch and wait“. Surg Oncol Clin North Am 1992; 1: 247-259

22 Morton DL, Wen DR, Wong JH et al. Technical details of intraoperative lymphatic mapping for early stage melanoma. Arch Surg 1992; 127: $392-399$

${ }^{23}$ Mudan A, Murray DR, Herda SC et al. Early stage melanoma: lymphoscintigraphy, reproducibility of sentinel node detection, and effectiveness of the intraoperative gamma probe. Radiology 1996; 199: $171-175$

${ }^{24}$ Orfanos CE, Jung EG, Garbe C, Rassner G. Stellungnahme und Empfehlungen der Kommission malignes Melanom der Deutschen Dermatologischen Gesellschaft zur Diagnostik, Behandlung und Nachsorge des malignen Melanoms der Haut. Hautarzt 1994; 45: 285-291

${ }^{25}$ Pijpers R, Borgstein PJ, Meijer S et al. Sentinel node biopsy in melanoma patients: Dynamic lymphoscintigraphy followed by intraoperative gamma probe and vital dye guidance. World J Surg 1997; 21: 788 - 793

${ }^{26}$ Reintgen D, Cruse CW, Wells K et al. The orderly progression of melanoma nodal metastases. Ann Surg 1994; 220: 759-767

${ }^{27}$ Ross MI. The case for elective lymphadenectomy. Surg Oncol Clin N Am 1992; 1: $205-222$

${ }^{28}$ Sim FH, Taylor WF, Pritchard DJ, Soule E. Lymphadenectomy in the management of stage I malignant melanoma: a prospective randomized study. Mayo Clin Proc 1986; 61: 697-705 
${ }^{29}$ Slingluff CL, Stidham KR, Ricci WM, Stanley WE, Seigler HF. Surgical management of regional lymph nodes in patients with melanoma. Ann Surg 1994; 219: 120-130

${ }^{30}$ Thompson JF, McCarthy WH, Bosch CMJ et al. Sentinel lymph node status as an indicator of the presence of metastatic melanoma in regional lymph nodes. Melanoma Res 1995; 5: 255-260

${ }^{31}$ Uren RF, Howman-Giles RB, Shaw HM, Thompson JF, McCarthy WH. Lymphoscintigraphy in high-risk melanoma of the trunk: predicting draining node groups, defining lymphatic channels and locating the sentinel node. J Nucl Med 1993; 34: 1435-1440
32 Urist MM, Maddox WA, Kennedy JE, Balch CM. Patient risk factors and surgical morbidity after regional lymphadenectomy in 204 melanoma patients. Cancer 1993; 51: 2152 - 2159

33 Veronesi U, Adamus J, Bandiera DC et al. Inefficacy of immediate node dissection in stage I melanoma of the limbs. N Engl J Med 1977; 297: $627-630$

${ }^{34}$ Vogt H, Wengenmair H, Kopp J, Dorn R, Gröber S, Heidenreich P. Der Sentinel-Lymphknoten (SLN): Prä- und intraoperative nuklearmedizinische Diagnostik. Der Nuklearmediziner 1999; 22: 233-242

35 Wengenmair H, Kopp J, Vogt H, Heidenreich P. Qualitätskriterien und Vergleich von Gammasonden zur Sentinel-Lymphonodektomie. Der Nuklearmediziner 1999; 22: $271-280$ 